\title{
Infección de Rattus rattus por Trypanosoma cruzi como indicador del control vectorial en Arequipa, Perú
}

\author{
Ayaqui, R.; Ruelas, N. \\ Depto. Microbiol. y Patología, Fac. Med. Univ. Nac. Arequipa, Perú. Presentado \\ al Congreso Lat. Parasitol. 2017, Santiago, Chile. E-mail: rayaqui@unsa.edu.pe
}

\begin{abstract}
Resumen
Ayaqui, R.; Ruelas, N.: Infección de Rattus rattus por Trypanosoma cruzi como indicador del control vectorial en Arequipa, Perú. Rev. Vet. 32: 1, 73-78, 2021. Como parte del programa de control de la enfermedad de Chagas en la región Arequipa, se vienen realizando rociados residuales, con deltametrina PM 5\%, y entre los criterios para evaluar la interrupción de la transmisión vectorial, no se considera la infección natural de los roedores sinantrópicos por T. cruzi. El objetivo fue determinar el índice de infección natural por T. cruzi en el roedor sinantrópico $R$. rattus, antes y después del rociado residual de las viviendas con deltametrina PM 5\%, como indicador del impacto del control vectorial de la enfermedad de Chagas. Se diseñó un estudio cuasi-experimental, a través de capturas de roedores en la etapa pre-rociado en el intradomicilio y capturas de roedores en la etapa post-rociado, en la localidad rural de Murco (Caylloma, Arequipa). Se les aplicó xenodiagnóstico por animal, con 10 ninfas III de Triatoma infestans. Los roedores fueron identificados como $R$. rattus y el índice de infección por el hemoflagelado identificado como T. cruzi, en el pre-rociado fue $84,2 \%$ y en el post-rociado fue $0 \%$. Además, en el pre-rociado el $50.0 \%$ (2/4) de Mus musculus fueron positivos a T. cruzi. Se concluye que la negativización de $R$. rattus en el post-rociado, indicaría la notable reducción de la transmisión o la interrupción de la transmisión vectorial de $T$. cruzi en la localidad de Murco.
\end{abstract}

Palabras clave: Trypanosoma cruzi, impacto, Rattus rattus, rociado, transmisión.

\begin{abstract}
Ayaqui, R.; Ruelas, N.: Rattus rattus infection by Trypanosoma cruzi as an indicator of vector control in Arequipa, Perú. Rev. Vet. 32: 1, 73-78, 2021. Residual insecticide spraying with deltamethrin PM 5\% has been a cornerstone strategy used in the Chagas disease Control Program in the region of Arequipa. Nonetheless, Trypanosoma cruzi natural infected synanthropic rodents are not used as an indicator to evaluate interruption of vector transmission. The objective was to determine the natural infection index by T. cruzi in the synanthropic rodent, Rattus rattus, before and after household residual insecticide spraying with deltamethrin PM 5\%, as impact indicator for Chagas disease vector control strategies. A quasi-experimental study was performed in the rural locality of Murco (Caylloma, Arequipa), through capture of rodents during household pre-spraying stage and capture of rodents during the post-spraying stage. One xenodiagnosis box was used in each animal containing 10 ny mphs III of Triatoma infestans. The rodent's identified was Rattus rattus and the infection index for the hemoflagellate identified as Trypanosoma cruzi, was $84.2 \%$ in the pre-spraying stage and $0 \%$ in the post-spraying stage. Furthermore, $50 \%(2 / 4)$ of Mus musculus were positive in the pre-spraying stage. It is concluded that the negativization of $R$. rattus in the post-spraying stage could indicate a remarkable transmission reduction or interruption of T. cruzi vector transmission in the locality of Murco.
\end{abstract}

Key words: Trypanosoma cruzi, impact, Rattus rattus, spray, transmission.

\section{INTRODUCCIÓN}

La enfermedad de Chagas es producida por el Trypanosoma cruzi y constituye un importante problema de salud pública ${ }^{9}$. Se transmite principalmente a través de las heces de insectos hematófagos de la fa- milia Reduviidae, por transfusión sanguínea, trasplante de órganos, transmisión vertical (congénita), alimentos contaminados y otras formas menos frecuentes ${ }^{5}$. La enfermedad de Chagas es una zoonosis cuyos reservorios son mamíferos domésticos, sinantrópicos y silvestres $8,9,17$

En las Américas se estima que existen, aproximadamente 6 millones de personas infectadas y entre 
28.000 a 30.000 casos agudos nuevos por año y otros 8000 casos por transmisión congénita ${ }^{24}$.

La enfermedad de Chagas ocasiona una merma de la productividad, debido a la mortalidad prematura de los pacientes y produce una pérdida de años de vida por discapacidad (AVAD) ${ }^{18}$. En la ciudad de Arequipa, durante el año 2000, se encontró que los índices epidemiológicos eran altos, así como la presencia de numerosos casos de la enfermedad de Chagas ${ }^{6,9}$.

Por tal razón, entre los años 2002 y 2003, el Ministerio de Salud de Perú, implementó un programa de control vectorial en la región Arequipa, diseñada en tres fases: una encuesta entomológica de base para determinar las áreas de intervención; una fase de "ataque" mediante dos aplicaciones anuales de insecticida residual deltametrina PM 5\% y un período continuo de vigilancia activa y pasiva para la detección de la infestación residual o re-infestaciones ${ }^{3,7,9}$.

Los estudios de seroprevalencia como línea de base del programa de control en población pre-escolar y escolar fue desde $11,3 \%$ hasta $12,5 \%{ }^{20}$; sin embargo, la prevalencia en los menores de 5 años podía llegar hasta $18,2 \%{ }^{21}$ y con los rociados disminuyeron progresivamente, hasta llegar entre $5,3 \%$ y $4,7 \%{ }^{19}$.

En el Perú, especialmente en la región de Arequipa, el cobayo es el reservorio doméstico más importante en la transmisión de T. cruzi ${ }^{1}$. En áreas rurales como el valle de Vítor; se han identificado otros reservorios sinantrópicos como Rattus rattus y Mus musculus infectados por T. cruzi, con prevalencias de infección más altas ${ }^{2}$ que los reservorios domésticos como Canis familiaris, Cavia porcellus, Feliscatus, Oryctolagus cuniculus y Sus scrofa domesticus ${ }^{9}$.

Los roedores sinantrópicos del género Rattus $s p$ son reservorios importantes en el ciclo de transmisión de T. cruzi, no solo por sus altas prevalencias de infección en el ambiente doméstico, sino también en el círculo silvestre, desempeñando un papel clave como amplificador y fuente de infección del parásito ${ }^{13,16,26,29}$. Por lo tanto, se debe evaluar su papel de reservorio y el de eslabón entre los ciclos doméstico y silvestre, de importancia epidemiológica ${ }^{27}$.
En un estudio previo en la localidad de Murco, distrito de Huanca, el índice de infestación domiciliaria (IID) fue $87,1 \%$ y el índice tripano-triatomino (ITT) fue $67,0 \%$. Además, los xenodiagnósticos aplicados a 39 personas fueron negativos a T. cruzi, pero fueron positivos dos cobayos ${ }^{9}$.

La localidad de Murco fue intervenida químicamente, y como se dispone de una medición basal en los roedores sinantrópicos del índice de infección natural por T. cruzi, es importante conocer el impacto del control vectorial.

Por ello, el objetivo del estudio fue determinar el índice de infección natural por T. cruzi en el roedor sinantrópico Rattus rattus, antes y después del rociado residual de las viviendas con deltametrina PM 5\%, como indicador del impacto del control vectorial de la enfermedad de Chagas en un área endémica de la región Arequipa, Perú.

\section{MATERIAL Y MÉTODOS}

Lugar del estudio. La localidad de Murco está a $2656 \mathrm{msnm}$, ubicada en $\left(16^{\circ} 04^{\prime} 48.45^{\prime \prime} \mathrm{LS}\right.$ y $\left.71^{\circ} 54 " 48.38^{\prime \prime} \mathrm{LO}\right)$ en el distrito de Huanca, provincia de Caylloma de la región Arequipa, en la zona sudoccidental del Perú (Figura 1). Es un valle interandino aislado, ubicado en la parte alta del río Siguas, con una temperatura que varía entre 10 y $21.7^{\circ} \mathrm{C}$. Es accesible por una carretera de trocha desde la ciudad de Arequipa. Las principales actividades son la agricultura con cultivos de maíz, trigo, habas, papa y alfalfa. La ganadería, con la cría de ganado bovino, porcino y ovino. Son unas 60 viviendas construidas principalmente de material rústico, con paredes de adobe, piso de tierra y techo de calamina o de paja.

Diseño del estudio. El diseño fue cuasi-experimental, no tuvo grupo control y se realizó una medición basal (índice de infección natural de roedores sinantrópicos por T. cruzi en la etapa pre-rociado), dos intervenciones -entre 2004 al 2010 - de las viviendas (rociado residual con deltametrina 5\% PM a $25 \mathrm{mg}$ i.a/ $\mathrm{m}^{2}$ ) por la Gerencia Regional de Salud de Arequipa, y

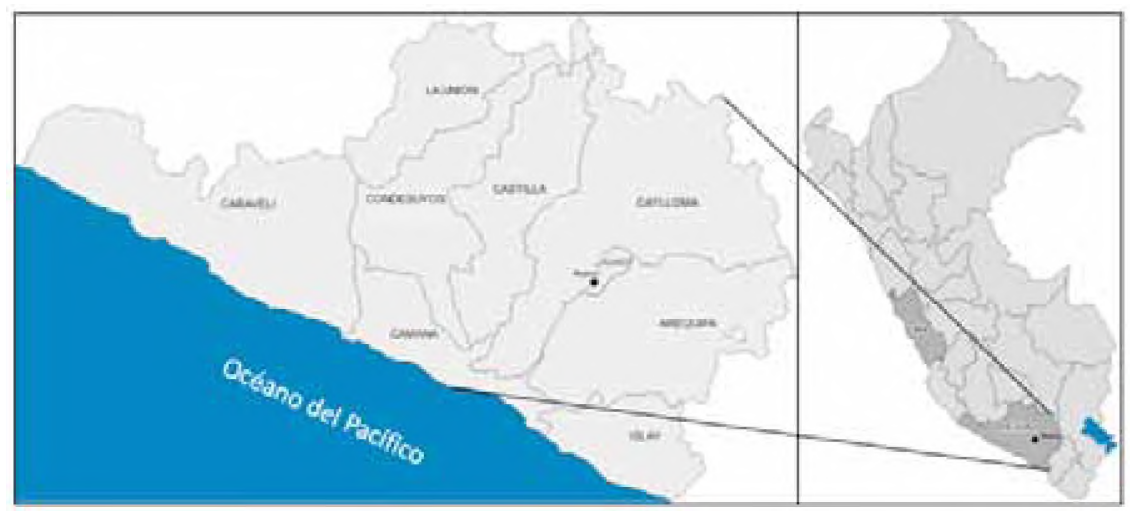

Figura 1. Mapa de ubicación de la localidad de Murco, distrito de Huanca, Provincia de Caylloma, Arequipa, Perú. 
luego una medición final (índice de infección natural de roedores sinantrópicos por $T$. cruzi en la etapa post-rociado).

Captura de roedores sinantrópicos. La captura de ratas se realizó en dos momentos: en la etapa prerociado en julio de 1998 por 7 días y en la etapa postrociado entre mayo y junio de 2012 por 10 días. Las trampas National modificadas fueron colocadas cebadas con cecina ahumada (carne seca) y queso, dentro de la vivienda donde había antecedentes de la presencia de ratas (cocina, dormitorio y almacén).

Luego fueron revisadas al día siguiente. Si eran positivas, la inmovilización de los roedores se realizó mediante la técnica de mareo ${ }^{2}$, que consiste en colocar la puerta de la trampa dentro de la boca de un saquillo de polietileno para facilitar la salida de la rata de la trampa al saquillo. Una vez aislada, se retira la trampa y se le da unas 5 vueltas al saquillo con la rata dentro, lo suficiente para marear al animal, pero sin dañarla.

Seguidamente, se aisló al roedor en un extremo del saquillo, se le tomó por el cuello y se dió vueltas al saquillo exponiendo la rata. Luego, fueron colocados los "nudos corredizos" a las patas con la ayuda de una pinza. Primero fueron aseguradas las patas delanteras y finalmente, la rata fue fijada a una tablilla.

En el caso de los ratones se utilizó trampa Sherman y se procedió de igual manera, con una bolsa de plástico transparente. La especie de roedores fue identificada en base a las medidas biométricas del cuerpo, cola y patas, además de su descripción morfológica de acuerdo a Gaviño y Juárez ${ }^{15}$.

Aplicación del xenodiagnóstico. A los roedores inmovilizados, se les aplicó en el campo una caja de xeno-diagnóstico con 10 ninfas III de Triatoma infestans para ratas, y con 6 para ratones; que estuvieron en ayunas por 20 días previos a su aplicación. Los triatominos fueron criados a $28^{\circ} \mathrm{C}$ y entre $70-80 \%$ de humedad relativa, alimentados con sangre de ave (gallo), en el insectario de la Facultad de Medicina de la Universidad Nacional San Agustín de Arequipa.

Finalmente, los roedores (ratas y ratones) fueron anestesiados con éter y muertos por dislocadura cervi$\mathrm{cal}^{23}$ y luego enterrados a un metro de profundidad, lejos de las viviendas. Las cajas de xenodiagnóstico aplicadas, fueron llevadas al insectario de la FM-UNSA, para su lectura a los 30 y 60 días, mediante el examen de las deyecciones de los triatominos ${ }^{1,2}$.

Para la identificación de T. cruzi, se siguieron las recomendaciones de Barreto ${ }^{4}$. Para ello, las ninfas positivas al hemoflagelado fueron alimentadas sobre un pollo, antes de proceder al "ordeño" para la obtención de los tripomastigotes metacíclicos. Una parte de estas formas fueron inoculadas en tubos, con el medio de cultivo Senejkie modificado, los que fueron mantenidos en estufa a $28^{\circ} \mathrm{C}$ y el control se realizó a los 7 días.

Las otras partes de las heces, fueron inoculadas a una rata Wistar, obtenida del bioterio de la FM-UNSA,
Tabla 1. Índice de infección natural de Rattus rattus por Trypanosoma cruzi en el pre y post-rociado, según el ambiente de captura, en la localidad de Murco.

\begin{tabular}{lcccccc}
\hline \multirow{2}{*}{$\begin{array}{l}\text { ambiente } \\
\text { de captura }\end{array}$} & \multicolumn{3}{c}{ pre-rociado } & 1998 & \multicolumn{3}{c}{ post-rociado 2012} \\
\cline { 2 - 7 } & total & \multicolumn{2}{c}{ infectados } & total & \multicolumn{2}{c}{ infectados } \\
& $\mathrm{N}^{\circ}$ & $\mathrm{N}^{\circ}$ & $\mathbf{\%}$ & $\mathrm{N}^{\circ}$ & $\mathrm{N}^{\circ}$ & $\%$ \\
\hline cocina & 7 & 7 & 100,0 & 2 & 0,0 & 0,0 \\
dormitorio & 22 & 19 & 86,4 & 10 & 0,0 & 0,0 \\
almacén & 9 & 6 & 66,7 & 2 & 0,0 & 0,0 \\
\hline total & 38 & 32 & 84,2 & 14 & 0,0 & 0,0 \\
\hline
\end{tabular}

vía intraperitoneal, la que fue evaluada a los 14 días mediante el examen directo de sangre y el xenodiagnóstico realizado con 10 ninfas III. La rata fue anestesiada con éter para ser sacrificada ${ }^{23}$.

Se realizaron frotis sanguíneos, que fueron coloreados con Giemsa para realizar la biometría de los parásitos, para ello se determinó la longitud del flagelo (LF), la del cuerpo (LC), la longitud total (LT), la del núcleo a la parte anterior (NA), de la parte posterior al núcleo (PN), y se calculó el índice nuclear medio (IN: $\mathrm{PN} / \mathrm{NA}$ ).

La biometría de T. cruzi se realizó con la forma tripomastigote sanguíneo en dibujos bajo una cámara Carl Zeiss a 100X, una porción de tejido del corazón, fue fijado en formol al $10 \%$ para realizar cortes histológicos de acuerdo a los procedimientos rutinarios, que fueron coloreados por hematoxilina-eosina, para la búsqueda de nidos de amastigotes de T. cruzi ${ }^{2}$.

Análisis de los datos. Se presentan las medias de las medidas biométricas de las ratas (longitud total, longitud de la cola y longitud de la pata), las medias con su desviación estándar (DE) de las medidas biométricas en $\mu \mathrm{m}$ del hemoflagelado y finalmente, se muestran en una tabla los índices de infección natural de las ratas por T. cruzi (pre y post-rociado) en frecuencia relativa y absoluta.

Aspectos éticos. En lo posible se evitó el sufrimiento de los roedores capturados en campo y la rata Wistar utilizada en la infección experimental de laboratorio, fue mantenida en condiciones adecuadas con comida y agua en una jaula individual en el bioterio de la FM-UNSA. Todos los roedores fueron anestesiados con éter y sacrificados de acuerdo a las recomendaciones de National Research Council ${ }^{23}$.

\section{RESULTADOS Y DISCUSIÓN}

Fueron capturadas 52 ratas, identificadas como Rattus rattus Linnaeus 1758. Los datos biométricos fueron: longitud total $39,5 \mathrm{~cm}$, longitud de la cola 21 $\mathrm{cm}$, longitud de la pata: $3,7 \mathrm{~cm}$. El índice de infección natural de $R$. rattus por $T$. cruzi (Tabla 1) en el prerociado fue de $84,2 \%(32 / 38)$ y el $50,0 \%$ (2/4) de $M u s$ musculus fueron positivos. El índice de infección de $R$. rattus post-rociado fue negativo $(0 / 14)$ 
El hemoflagelado procedente del pre-rociado de $R$. rattus, en el frotis sanguíneo fue fusiforme, en forma de "C" o "S", con la membrana ondulante recorriendo casi todo el cuerpo, con el kinetoplasto subterminal. Las medidas biométricas fueron LT: $21,7 \mathrm{DE} 2,4 \mu \mathrm{m}$, LC: 14,9 DE $1,3 \mu \mathrm{m}, \mathrm{LF}: 6 \mathrm{DE} 1,0 \mu \mathrm{m}$, L núcleo: 1,9 DE $0,2 \mu \mathrm{m}$, PN: 6,9 DE $0,6 \mu \mathrm{m}$, NA: 5,9 DE 1,0 $\mu \mathrm{m}$. El IN fue de 1,1 .

La inoculación en el medio de cultivo Senejkie modificado, fue positivo a los siete días, definido por la reproducción de epimastigotes en forma de rosetas. La rata Wistar infectada experimentalmente, fue positiva a los 14 días con la presencia de tripomastigotes sanguíneos.

También, fueron positivas al xenodiagnóstico aplicado, las ratas capturadas en campo y la rata Wistar infectada experimentalmente. En base a estos resultados, el hemoflagelado fue identificado como Trypanosoma cruzi Chagas, 1909.

Murco es una localidad rural endémica de la enfermedad de Chagas; en el pre-rociado el índice de infección natural por $T$. cruzi en especímenes de $R$. rattus de $84,2 \%$, es uno de los índices más altos reportados para la región y para América Latina en roedores sinantrópicos.

Este valor es más bajo que el $100 \%$ de infección natural en Bahía, Brasil ${ }^{16}$, pero es similar al $83.6 \%$ en la misma especie en Coquimbo, Chile ${ }^{29}$. Sin embargo, es más alto que el $38.5 \%$ en Bolivia ${ }^{16}$, al $29,41 \%$ por serología en Mexico ${ }^{30} \mathrm{y}$ al $36.1 \%$ en el valle de Vítor. Arequipa ${ }^{2}$. Este alto índice de infección natural por $T$. cruzi de $R$. rattus en el pre-rociado se explica por los altos índices de IID de $87,1 \%$ e ITT de $67,0 \%$ del área de estudio ${ }^{9}$.

En el pre-rociado, también se encontró otro reservorio positivo, el M. musculus con 50,0\% (2/4); este valor está por encima del $38,8 \%$ en el valle de Vítor, Arequipa $^{2}$, pero por debajo del $83,3 \%$ de la región de Coquimbo en Chile ${ }^{29}$. Este es el primer estudio en el Perú, que evalúa el impacto de la transmisión vectorial a través de roedores sinantrópicos en un área donde se distribuye T. infestans.

El índice de infección de $R$. rattus en Murco fue alto en el pre-rociado, de $84,2 \%$, lo que indica una transmisión vectorial dinámica de $T$. cruzi a los roedores sinantrópicos; mientras que en el post-rociado fue negativo (0/14), lo que indica la interrupción de la transmisión de T. cruzi por Triatoma infestans o la notable reducción de la transmisión a niveles no detectables debido al rociado residual. Sin embargo, sería recomendable evaluar a los animales domésticos, para tener una idea más clara del impacto del control químico en la transmisión vectorial del T. cruzi.

Es importante mencionar que en Chile, mediante la técnica PCR, se demostró la presencia en viviendas rurales de varios clones de $T$. cruzi en T. infestans positivos y en pacientes con el T. cruzi clone 19 (TCI) de origen silvestre ${ }^{10}$.
Este mismo $T c I$ ha sido encontrado en la zona de Arequipa ${ }^{25}$. Sin duda, esta situación puede repetirse en la región sur del Perú, debido a la presión selectiva del insecticida contra el único vector domiciliario, el $T$. infestans, por lo cual se recomienda como un indicador adicional para evaluar la interrupción vectorial en las zonas rurales, el índice de infección de reservorios sinantrópicos y domésticos; mientras que para el área urbana la infección es en reservorios domésticos.

Existen varias ventajas al utilizar el índice de infección natural de los reservorios como un indicador de impacto vectorial en el sur del Perú. Por ejemplo, se pueden medir en un tiempo más corto que la serología contra anticuerpos específicos de T. cruzi en menores de 0 a 5 años; además, en las zonas rurales, os niños menores de 5 años pueden representar un problema operativo para el personal de salud.

R. rattus es uno de los principales reservorios sinantrópicos de T. cruzi en las regiones de Arequipa ${ }^{2}$, Venezuela ${ }^{11,14}$ y Chile ${ }^{29}$ Estos hallazgos confirman su importancia como reservorio y fuente potencial de ingreso de T. cruzi al ciclo doméstico, por su capacidad de invadir el domicilio y como indicador importante de la dinámica del T. cruzi entre el ciclo silvestre, peridoméstico y doméstico ${ }^{16}$.

En Murco, además del impacto del rociado, es probable que el mejoramiento de las viviendas puede haber influido en la reducción de la transmisión de $T$. cruzi. En la etapa pre-rociado, la mayoría tenían techo de paja, lo cual es favorable para el vector y los roedores sinantrópicos; sin embargo, en la etapa post-rociado, los techos habían sido cambiados por calaminas, que no ofrecen condiciones favorables para el vector y para los roedores; lo que también podría haber influido en la menor captura de $R$. rattus.

La identificación de T. cruzi (Chagas, 1909) se realizó siguiendo los criterios de Barreto ${ }^{4}$, cuyos resultados son similares a los descritos en $R$. rattus del valle de Vítor, Arequipa ${ }^{2}$. Sin embargo, el aspecto que refuerza nuestros hallazgos fue la formación de los nidos de amastigotes en las fibras cardíacas de la rata Wistar infectada experimentalmente, pues solo T. cruzi se reproduce intracelularmente ${ }^{4}$. En este estudio no se observaron flagelados en la hemolinfa de los especímenes de $T$. infestans con xenodiagnósticos positivos para $T$. cruzi, lo cual concuerda con la ausencia de T. rangeli en la región sudoccidental del Perú.

El control químico es la principal herramienta para la interrupción de la transmisión vectorial de la enfermedad de Chagas en el Perú, el cual ha permitido lograr esa meta en Tacna ${ }^{28}$. En la región del Chaco Argentino se ha postulado que el hallazgo de colonias silvestres de $T$. infestans podría estar asociado a la presión selectiva del control químico, y que estos focos representarían un riesgo como fuente para la reintroducción de la transmisión doméstica de T. $_{\text {cruzi }}{ }^{9}$.

Otro aspecto que refuerza la necesidad de estudiar mejor la dinámica de transmisión y la interacción entre los reservorios animales, el vector y el hombre, es que 
la enfermedad de Chagas se ha urbanizado en la ciudad de Arequipa, debido a la migración de la población desde zonas rurales ${ }^{9,22}$.

En el contexto de las acciones del Programa de Control de la Enfermedad de Chagas en la Región Arequipa, en las áreas con aplicación de insecticidas residuales se recomienda mantener la vigilancia postrociado, para no poner en riesgo los logros alcanzados, tal como ocurrió entre los años 1970-1972 ${ }^{12}$, que luego del programa de control del vector, se suspendió la vigilancia, lo que ocasionó el incremento del vector y su dispersión geográfica hacia otros valles y a la ciudad de Arequipa, logrando alcanzar niveles de infestación e infección más altos que la medición basal antes de las intervenciones ${ }^{1,20,21}$.

Entre las limitaciones de este estudio, en el post-rociado están el bajo número de roedores capturados y el no haber identificado el linaje de T. cruzi; sin embargo, es un estudio muy importante en un área con control químico, para evitar la presencia de reservorios en la vivienda y alcanzar el objetivo de interrumpir la transmisión intradomiciliaria de la enfermedad de Chagas ${ }^{13}$.

En este estudio, el índice de infección de Rattus rattus por T. cruzi en el pre-rociado fue de $84,2 \%$ y en el post-rociado fue de $0 \%$, además, en el pre-rociado el $50,0 \%(2 / 4)$ de $M$. musculus fueron positivos a T. cruzi. La negativización de $R$. rattus en el post-rociado, indicaría la notable reducción de la transmisión o la interrupción de la transmisión vectorial de $T$. cruzi en la localidad de Murco.

Agradecimientos. A la Universidad Nacional de San Agustín de Arequipa, por el financiamiento para la presentación del trabajo en el XXIV Congreso Latinoamericano de Parasitología, Santiago de Chile, 2017. Al Técnico Sr. C. N. Calsin por su colaboración en el trabajo de campo. A las autoridades del MINSA y de la localidad de Murco por las facilidades brindadas.

\section{REFERENCIAS}

1. Ayaqui R, Córdova E. 1988. Epidemiología de la enfermedad de Chagas en el valle de Vítor (Distrito de Vítor, Dpto. Arequipa). Bol Peru Parasitol 4: 19-23.

2. Ayaqui R, Córdova E. 1990. Infección natural de roedores sinantrópicos por Trypanosoma cruzi (Chagas-1909) en el valle del río Vitor (Arequipa-Perú). Acta Méd Agust 1: 66-70.

3. Barbu CM et al. 2014. Residual infestation and recolonization during urban Triatoma infestans Bug Control Campaign, Peru. Emerg Infect Dis 20: 2055-2063.

4. Barreto MP. 1979. Reservatorios do Trypanosoma (Schizotrypanum) cruzi, 1909. In: Anais do Simposio sobre Molestia da Chagas, Sao Paulo, Brasil.

5. Bern C. 2015. Chagas' disease. N Engl JMed 73: 456-466.

6. Bowman NM et al. 2008. Chagas disease transmission in periu rban communities of Arequipa, Peru. Clin Infect Dis 46: $1822-1828$.
7. Buttenheim AM et al. 2019. A behavioral design approach to improving a Chagas disease vector control campaign in Peru. BMC Public Health 9: 1272.

8. Ceballos LA et al. 2011. Hidden sylvatic foci of the main vector of Chagas disease Triatoma infestans: threats to the vector elimination campaign? PLoSNegl Trop Dis 5(10): e- 1365 .

9. Córdova E. 2005. Triatoma infestans y la enfermedad de Chagas en el sur del Perú, 1917-2004. Tesis Doctoral, Univ. Nacional San Agustín de Arequipa, p. 1-56.

10. Coronado X et al. 2006. Variation in Trypanosoma cruzi clonal composition detected in blood patients and xenodiagnosis triatomines: implications in the molecular epidemiology of Chile. Am J Trop Med Hyg 74: 1008-1012.

11. Delima H. et al. 2006. Try panosomatidae de importancia en salud pública en animales silvestres y sinantrópicos en un área rural del municipio Tovar del estado Mérida, Venezuela. Biomédica 26: 42-50.

12. Flores M. 1978. Control de triatominos con hexaclorociclohexano en tres departamentos del Perú. Bol Of San Pan 84:324-329.

13. Flores A. et al. 2019. Trypanosoma cruzi transmission dynamics in a synanthropic and domesticated host community. PLoSNegl Trop Dis13;13(12): e0007902.

14. García HA et al. 2019. Zoonotic trypanosomes in rats and fleas of venezuelan slums. Ecohealth 6: 523-533.

15. Gaviño C, Juárez C, Figueroa M. 2004. Técnicas biológicas selectas de laboratorio y de campo, 6ta ed., Editorial Limusa, México DF.

16. Gürtler RE, Cardinal MV. 2015. Reservoir host competence and the role of domestic and commensal hosts in the transmission of Trypanosoma cruzi. Acta Trop 151: 32-50.

17. Hernández CI et al. 2018. Frequency of Trypanosoma cruzi infection in synanthropic and wild rodents captured in a rural community in southeast of Mexico. Vet Med Int 2018: 8059613 .

18. Lee BY, Bacon KM, Bottazzi ME, Hotez PJ. 2013. Global economic burden of Chagas disease: a computational simulation model. Lancet Infect Dis 13: 342-348.

19. Levy MZ et al. 2007. Targeted screening strategies to detect Trypanosoma cruzi infection in children. PLoSNegl Trop Dis 1(3):e103.

20. Liu M et al. 2012. Estudio epidemiológico de la enfermedad de Chagas en el Pueblo Joven San Pedro de Tiabaya, Arequipa, Perú. Acta Med UNSA 1: 57-64.

21. Mendoza $\mathbf{C}$ et al. 2005. Seroprevalencia de la infección por Trypanosoma cruzi en escolares del valle de Vítor, Arequipa, Perú. Diagnóstico 44: 111-115.

22. Náquira C. 2014. Urbanización de la enfermedad de Chagas en el Perú: experiencias en su prevención y control. RevPeruMedExp Salud Publica 31(2):343-347.

23. National Research Council. 1996. Guide for the Care and Use of Laboratory Animals. Washington, DC: National Academy Press

24. Organización Panamericana de Salud (OPS). Guía para el diagnóstico y el tratamiento de la enfermedad de Chagas. Washington, D.C.: Organización Panamericana de la Salud; 2018. https://iris.paho.org/bitstream/handle.spa.pdf 
25. Padilla C. 2017. Detección de unidades discretas de tipificación de Trypanosoma cruzi en triatominos recolectados en diferentes regiones naturales de Perú. Biomédica 37:167-179.

26. Rosal GG et al. 2018. Chagas disease: Importance of rat as reservoir hosts of Trypa nosoma cruzi (Chagas, 1909) in western Mexico. J Infect Publ Health 11: 230-233.

27. Rozas M et al. 2005. Trypanosoma cruzi infection in wild mammals from a chaga-sic area of Chile. Am J Trop Med Hyg 73: 517-519.
28. Tejada E, Villanueva J. 2011. Certificación de la interrupción de la transmisión vectorial de la enfermedad de Chagas en áreas endémicas de Tacna, Perú. Rev Peru Epidemiol 15: 35-41.

29. Yefi QE et al. 2018. Trypanosoma cruzi load in synanthropic rodents from rural areas in Chile. Parasit Vectors 11: 171.

30. Zavala VJ et al. 1996. Infection by Trypanosoma cruzi in mammals in Yucatan, Mexico: a serological and parasitological study. Rev Inst Med Trop Sao Paulo 38: 289-292.

\section{Revista Veterinaria ingresa a SciELO}

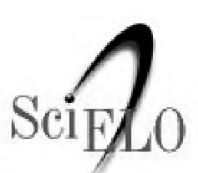

Scientific Electronic Library Online

Revista Veterinaria, publicación oficial de la Facultad de Ciencias Veterinarias de la Universidad Nacional del Nordeste (Corrientes, Argentina), ha logrado acceder al Núcleo Básico de Revistas Científicas Argentinas (Nivel 1), luego de calificar adecuadamente en el Centro Argentino de Información Científica y Tecnológica (CAICYT), según Resolución 2485/14 del Consejo Nacional de Investigaciones Científicas y Técnicas (CONICET).

Sobre un puntaje máximo de 33 se obtuvieron 32 puntos. Tal calificación constituye "una garantía de la excelencia de la publicación" (sic) y queda expedita la vía del Portal SciELO (Scientific Electronic Library Online) para los artículos publicados.

En tal calificación gravitó positivamente la circunstancia de haber aumentado el índice de impacto (Scimago-Elsevier) y haber disminuido las autocitaciones. También se tuvieron en cuenta aspectos como la amplia cobertura de la revista, la calidad científica del Comité Editorial, los criterios de evaluación de los artículos, el origen de los autores (locales $60 \%$, nacionales $13 \%$, extranjeros $24 \%$, en idioma inglés), el adecuado balance entre trabajos científicos originales y reseñas bibliográficas (ambos con alta calidad), así como el estricto cumplimiento de la periodicidad semestral y la favorable acogida por indizadores como Cab, J-Gate, Doaj, Ebsco, Gale Cengage, Infocyt, Latindex y Scopus.

Se consolida de esta manera la continuidad de "Revista Veterinaria", que en su acontecer registra más de 50 años de existencia en nuestra Facultad de Ciencias Veterinarias, entidad que en 2019 cumplió el $99^{\circ}$ aniversario de su fundación. 